\title{
Logarithmically complete monotonicity of a function related to the Catalan-Qi function
}

\author{
Feng Qi \\ Institute of Mathematics, \\ Henan Polytechnic University, China \\ College of Mathematics, \\ Inner Mongolia University for \\ Nationalities, China \\ Department of Mathematics, \\ College of Science, \\ Tianjin Polytechnic University, China \\ email: qifeng618@gmail.com
}

\author{
Bai -Ni Guo \\ School of Mathematics and Informatics, \\ Henan Polytechnic University, China \\ email: bai.ni.guo@gmail.com
}

\begin{abstract}
In the paper, the authors find necessary and sufficient conditions such that a function related to the Catalan-Qi function, which is an alternative generalization of the Catalan numbers, is logarithmically complete monotonic.
\end{abstract}

\section{Introduction}

It is stated in $[11,40]$ that the Catalan numbers $C_{n}$ for $n \geq 0$ form a sequence of natural numbers that occur in tree enumeration problems such as "In how many ways can a regular $n$-gon be divided into $n-2$ triangles if different orientations are counted separately?" whose solution is the Catalan number $C_{n-2}$. The Catalan numbers $C_{n}$ can be generated by

2010 Mathematics Subject Classification: 11B75, 11B83, 11Y35, 11Y55, 11Y60, 26A48, 33B15

Key words and phrases: necessary and sufficient condition, logarithmically complete monotonicity, Catalan number, Catalan-Qi function 


$$
\frac{2}{1+\sqrt{1-4 x}}=\frac{1-\sqrt{1-4 x}}{2 x}=\sum_{n=0}^{\infty} C_{n} x^{n}=1+x+2 x^{2}+5 x^{3}+14 x^{4}+\cdots .
$$

One of explicit formulas of $C_{n}$ for $n \geq 0$ reads that

$$
C_{n}=\frac{4^{n} \Gamma(n+1 / 2)}{\sqrt{\pi} \Gamma(n+2)},
$$

where

$$
\Gamma(z)=\int_{0}^{\infty} t^{z-1} e^{-t} d t, \quad \Re(z)>0
$$

is the classical Euler gamma function. In [8, 11, 40, 43], it was mentioned that there exists an asymptotic expansion

$$
\mathrm{C}_{x} \sim \frac{4^{\mathrm{x}}}{\sqrt{\pi}}\left(\frac{1}{\chi^{3 / 2}}-\frac{9}{8} \frac{1}{\chi^{5 / 2}}+\frac{145}{128} \frac{1}{\chi^{7 / 2}}+\cdots\right)
$$

for the Catalan function $\mathrm{C}_{x}$.

A generalization of the Catalan numbers $C_{n}$ was defined in $[9,10,16]$ by

$$
p d_{n}=\frac{1}{n}\left(\begin{array}{c}
p n \\
n-1
\end{array}\right)=\frac{1}{(p-1) n+1}\left(\begin{array}{c}
p n \\
n
\end{array}\right)
$$

for $n \geq 1$. The usual Catalan numbers $C_{n}={ }_{2} d_{n}$ are a special case with $p=2$.

In combinatorics and statistics, the Fuss-Catalan numbers $A_{n}(p, r)$ are defined $[6,45]$ as numbers of the form

$$
A_{n}(p, r)=\frac{r}{n p+r}\left(\begin{array}{c}
n p+r \\
n
\end{array}\right)=r \frac{\Gamma(n p+r)}{\Gamma(n+1) \Gamma(n(p-1)+r+1)} .
$$

It is easy to see that

$$
A_{n}(2,1)=C_{n}, \quad n \geq 0 \quad \text { and } \quad A_{n-1}(p, p)={ }_{p} d_{n}, \quad n \geq 1 .
$$

There have existed some literature, such as $[2,4,5,7,12,14,18,19,20,21$, $41,42,45]$, on the investigation of the Fuss-Catalan numbers $A_{n}(p, r)$.

In [31, Remark 1], an alternative and analytical generalization of the Catalan numbers $C_{n}$ and the Catalan function $C_{x}$ was introduced by

$$
\mathrm{C}(\mathrm{a}, \mathrm{b} ; z)=\frac{\Gamma(\mathrm{b})}{\Gamma(\mathrm{a})}\left(\frac{\mathrm{b}}{\mathrm{a}}\right)^{z} \frac{\Gamma(z+\mathrm{a})}{\Gamma(z+\mathrm{b})}, \quad \mathfrak{R}(\mathrm{a}), \mathfrak{R}(\mathrm{b})>0, \quad \mathfrak{R}(z) \geq 0 .
$$

For the uniqueness and convenience of referring to the quantity $C(a, b ; x)$, we call the quantity $\mathrm{C}(\mathrm{a}, \mathrm{b} ; \mathrm{x})$ the Catalan-Qi function and, when taking 
$\mathrm{x}=\mathrm{n} \geq 0$, call $\mathrm{C}(\mathrm{a}, \mathrm{b} ; \mathrm{n})$ the Catalan-Qi numbers. In the recent papers $[13$, $15,22,24,25,29,30,31,32,33,34,39]$, among other things, some properties, including the general expression and a generalization of the asymptotic expansion (1), the monotonicity, logarithmic convexity, (logarithmically) complete monotonicity, minimality, Schur-convexity, product and determinantal inequalities, exponential representations, integral representations, a generating function, connections with the Bessel polynomials and the Bell polynomials of the second kind, and identities, of the Catalan numbers $C_{n}$, the Catalan function $C_{x}$, the Catalan-Qi numbers $C(a, b ; n)$, the Catalan-Qi function $C(a, b ; x)$, and the Fuss-Catalan numbers $A_{n}(p, r)$ were established. Very recently, we discovered in [25, Theorem 1.1] a relation between the Fuss-Catalan numbers $A_{n}(p, r)$ and the Catalan-Qi numbers $C(a, b ; n)$, which reads that

$$
A_{n}(p, r)=r^{n} \frac{\prod_{k=1}^{p} C\left(\frac{k+r-1}{p}, 1 ; n\right)}{\prod_{k=1}^{p-1} C\left(\frac{k+r}{p-1}, 1 ; n\right)}
$$

for integers $n \geq 0, p>1$, and $r>0$.

Recall from $[3,26,28,38]$ that an infinitely differentiable and positive function $\mathrm{f}$ is said to be logarithmically completely monotonic on an interval $\mathrm{I}$ if it satisfies $0 \leq(-1)^{k}[\ln f(x)]^{(k)}<\infty$ on I for all $k \in \mathbb{N}$.

From the viewpoint of analysis, motivated by the idea in the papers [27, $35,36,37]$ and closely-related references cited therein, the author considered in [23] the function $\mathcal{C}_{a, b ; x}(t)=C(a+t, b+t ; x)$ for $t, x \geq 0$ and $a, b>0$ and obtained the following conclusions:

1. the function $\mathcal{C}_{a, b ; x}(t)$ is logarithmically completely monotonic on $[0, \infty)$ if and only if either $0 \leq x \leq 1$ and $a \leq b$ or $x \geq 1$ and $a \geq b$,

2. the function $\frac{1}{\mathrm{e}_{\mathrm{a}, \mathrm{b} ; \mathrm{x}}(\mathrm{t})}$ is logarithmically completely monotonic on $[0, \infty)$ if and only if either $0 \leq x \leq 1$ and $a \geq b$ or $x \geq 1$ and $a \leq b$.

This implies the logarithmically complete monotonicity of $\left[\mathcal{C}_{a, b ; x}(t)\right]^{ \pm 1}$ in $t \geq 0$ along with the ray $\left\{\begin{array}{l}u(t)=a+t \\ v(t)=b+t\end{array}\right.$ on the plane $(u, v)$, where $x \geq 0$ and $a, b>0$. Then one may ask a question: how about its logarithmically complete monotonicity along the ray $\left\{\begin{array}{l}u(t)=a+\alpha t \\ v(t)=b+\beta t\end{array}\right.$ for $\alpha, \beta \geq 0$ with $(\alpha, \beta) \neq(0,0)$ when $x, t \geq 0$ and $a, b>0$ ? In other words, is the function

$$
\mathcal{C}_{a, b ; x ; \alpha, \beta}(t)=C(a+\alpha t, b+\beta t ; x), \quad x \geq 0, \quad a, b>0
$$


of logarithmically complete monotonicity in $t \in[0, \infty)$ ? When $\alpha=\beta \neq 0$, this question has been answered essentially by the above-mentioned conclusions in [23]; when $\alpha=0$ or $\beta=0$, this question has been answered virtually by [34, Theorem 1.2] which states that the function $[\mathrm{C}(\mathrm{a}, \mathrm{b} ; \mathrm{x})]^{ \pm 1}$ is logarithmically completely monotonic

1. with respect to $a>0$ if and only if $x \gtrless 1$,

2. with respect to $b>0$ if and only if $x \lessgtr 1$.

In this paper, we will discuss the rest cases $\alpha, \beta>0$ and $\alpha \neq \beta$ of the above question. Our main results can be formulated as the following theorem.

Theorem 1 If and only if $\alpha=0$ and $\beta>0$, or $\alpha>0$ and $\beta=0$, or $\alpha=\beta>$ 0 , the function $\mathcal{C}_{\mathrm{a}, b ; x ; \alpha, \beta}(\mathrm{t})$ is of some logarithmically complete monotonicity. Concretely speaking,

1. the function $[\mathrm{C}(\mathrm{a}, \mathrm{b} ; \mathrm{x})]^{ \pm 1}$ is logarithmically completely monotonic

(a) with respect to $\mathrm{a}>0$ if and only if $\mathrm{x} \gtrless 1$,

(b) with respect to $\mathrm{b}>0$ if and only if $\mathrm{x} \lessgtr 1$,

2. the function $\mathcal{C}_{\mathrm{a}, \mathrm{b} ; \mathrm{x}}(\mathrm{t})$ is logarithmically completely monotonic on $[0, \infty)$ if and only if either $0 \leq x \leq 1$ and $\mathrm{a} \leq \mathrm{b}$ or $\mathrm{x} \geq 1$ and $\mathrm{a} \geq \mathrm{b}$,

3. the function $\frac{1}{\mathrm{e}_{\mathrm{a}, \mathrm{b} ; \mathrm{x}}(\mathrm{t})}$ is logarithmically completely monotonic on $[0, \infty)$ if and only if either $0 \leq x \leq 1$ and $\mathrm{a} \geq \mathrm{b}$ or $\mathrm{x} \geq 1$ and $\mathrm{a} \leq \mathrm{b}$.

\section{Proof of Theorem 1}

Taking the logarithm of $\mathcal{C}_{a, b ; x ; \alpha, \beta}(t)$ and differentiating with respect to $t$ give

$$
\begin{aligned}
{\left[\ln \mathcal{C}_{a, b ; x ; \alpha, \beta}(t)\right]^{\prime}=} & \psi(\beta t+b)-\psi(\alpha t+a)+x\left(\frac{1}{\beta t+b}-\frac{1}{\alpha t+a}\right) \\
& +\psi(\alpha t+x+a)-\psi(\beta t+x+b) .
\end{aligned}
$$

Making use of

$$
\psi(z)=\int_{0}^{\infty}\left(\frac{e^{-\mathfrak{u}}}{\mathfrak{u}}-\frac{e^{-z \mathfrak{u}}}{1-e^{-\mathfrak{u}}}\right) \mathrm{d} u, \quad \mathfrak{R}(z)>0
$$


in $[1$, p. 259, 6.3.21] leads to

$$
\begin{aligned}
& {\left[\ln \mathcal{C}_{a, b ; x ; \alpha, \beta}(t)\right]^{\prime}=\int_{0}^{\infty} \frac{e^{-(a+\alpha t) u}-e^{-(b+\beta t) u}}{1-e^{-u}} d u} \\
& +x \int_{0}^{\infty}\left[e^{-(b+\beta t) u}-e^{-(a+\alpha t) u}\right] d u \\
& +\int_{0}^{\infty} \frac{e^{-(b+\beta t) u}-e^{-(a+\alpha t) u}}{1-e^{-u}} e^{-\chi u} d u \\
& =\int_{0}^{\infty}\left[e^{-\chi \mathfrak{u}}-1+x\left(1-e^{-\mathfrak{u}}\right)\right] \frac{e^{-(b+\beta t) u}-e^{-(a+\alpha t) u}}{1-e^{-\mathfrak{u}}} \mathrm{d} u \\
& =x \int_{0}^{\infty}\left(\frac{1-e^{-\mathfrak{u}}}{\mathfrak{u}}-\frac{1-e^{-\chi \mathfrak{u}}}{x \mathfrak{u}}\right) \frac{e^{-(b+\beta t) u}-e^{-(a+\alpha t) u}}{1-e^{-\mathfrak{u}}} \mathfrak{u} \mathrm{d} u .
\end{aligned}
$$

It is easy to see that the function $\frac{1-e^{-\mathfrak{u}}}{\mathfrak{u}}$ is positive and strictly decreasing on $(0, \infty)$. Hence,

$$
\frac{1-e^{-\mathfrak{u}}}{\mathfrak{u}}-\frac{1-e^{-x u}}{x \mathfrak{u}} \gtreqless 0
$$

for $u \in(0, \infty)$ if and only if $x \lesseqgtr 1$.

Recall from [17, Chapter XIII], [38, Chapter 1], and [44, Chapter IV] that an infinitely differentiable function $f$ is said to be completely monotonic on an interval $\mathrm{I}$ if it satisfies $0 \leq(-1)^{\mathrm{k}} \mathrm{f}^{(\mathrm{k})}(\mathrm{x})<\infty$ on I for all $k \geq 0$. It is not difficult to see that a positive function $f$ is logarithmically completely monotonic if and only if the function $-(\ln f)^{\prime}$ is completely monotonic. The famous Bernstein-Widder theorem, [44, p. 160, Theorem 12a], states that a necessary and sufficient condition that $f(x)$ should be completely monotonic in $0 \leq x<\infty$ is that $f(x)=\int_{0}^{\infty} e^{-x t} d \alpha(t)$, where $\alpha$ is bounded and nondecreasing and the above integral converges for $0 \leq x<\infty$. Therefore, it is sufficient to find necessary and sufficient conditions on $a, b>0$ and $\alpha, \beta>0$ with $\alpha \neq \beta$ for the function

$$
\begin{aligned}
& e^{-(b+\beta t) u}-e^{-(a+\alpha t) u}=\int_{(b+\beta t) u}^{(a+\alpha t) u} e^{-v} d v \\
& =\int_{0}^{1}[(a-b)+(\alpha-\beta) t] u e^{-[(1-s)(b+\beta t)+s(a+\alpha t)] u} d s \\
& =\int_{0}^{1}[(a-b)+(\alpha-\beta) t] e^{-[(1-s) \beta+s \alpha] u t} u e^{-[(1-s) b+s a] u} d s
\end{aligned}
$$

to be completely monotonic in $t \in[0, \infty)$ for all $u \in(0, \infty)$. 
By induction, we obtain

$$
\left[(\mathrm{A}+\mathrm{Bt}) e^{-\mathrm{D} t}\right]^{(\mathrm{k})}=(-1)^{\mathrm{k}} \mathrm{D}^{\mathrm{k}-1}(\mathrm{BDt}+\mathrm{AD}-\mathrm{kB}) e^{-\mathrm{Dt}}, \quad \mathrm{k} \geq 0,
$$

where $A, B, D$ are real constants. Accordingly, the function $(A+B t) e^{-D t}$ is completely monotonic in $t \in[0, \infty)$ if and only if $A, B \geq 0, D>0$, and

$$
D^{k-1}(B D t+A D-k B) \geq 0, \quad k \geq 0, \quad t \in[0, \infty) .
$$

Simply speaking, the function $(A+B t) e^{-D t}$ is completely monotonic in $t \in$ $[0, \infty)$ if and only if $A \geq 0, B=0$, and $D>0$. Applying $A$ to $a-b, B$ to $\alpha-\beta$, and $D$ to $[(1-s) \beta+s \alpha] u$ yields that the function $e^{-(b+\beta t) u}-e^{-(a+\alpha t) u}$ is completely monotonic in $t \in[0, \infty)$ if and only if $a \geq b, \alpha=\beta$, and $\alpha, \beta \geq 0$ with $(\alpha, \beta) \neq(0,0)$. Combining this result with the inequality (2) and with the proofs of [23, Theorem 1.1] and [34, Theorem 1.2] concludes that, if and only if $\alpha=0$ and $\beta>0$, or $\alpha>0$ and $\beta=0$, or $\alpha=\beta>0$, the function $\mathcal{C}_{a, b ; x ; \alpha, \beta}(t)$ is of some logarithmically complete monotonicity. The proof of Theorem 1 is thus complete.

\section{References}

[1] M. Abramowitz, I. A. Stegun (Eds), Handbook of Mathematical Functions with Formulas, Graphs, and Mathematical Tables, National Bureau of Standards, Applied Mathematics Series 55, 10th printing, Washington, 1972.

[2] N. Alexeev, F. Götze, A. Tikhomirov, Asymptotic distribution of singular values of powers of random matrices, Lith. Math. J., 50 (2010), 121-132; Available online at http://dx.doi.org/10.1007/s10986-010-9074-4.

[3] R. D. Atanassov, U. V. Tsoukrovski, Some properties of a class of logarithmically completely monotonic functions, C. R. Acad. Bulgare Sci., 41 (1988), 21-23.

[4] J. -C. Aval, Multivariate Fuss-Catalan numbers, Discrete Math., 308 (2008), 4660-4669; Available online at http://dx.doi.org/10.1016/j. disc.2007.08.100.

[5] D. Bisch, V. Jones, Algebras associated to intermediate subfactors, Invent. Math., 128 (1997), 89-157; Available online at http://dx.doi. org/10.1007/s002220050137. 
[6] N. I. Fuss, Solutio quaestionis, quot modis polygonum n laterum in polygona m laterum, per diagonales resolvi queat, Nova Acta Academiae Sci. Petropolitanae, 9 (1791), 243-251.

[7] I. G. Gordon, S. Griffeth, Catalan numbers for complex reflection groups, Amer. J. Math., 134 (2012), 1491-1502; Available online at http://dx. doi.org/10.1353/ajm.2012.0047.

[8] R. L. Graham, D. E. Knuth, O. Patashnik, Concrete Mathematics-A Foundation for Computer Science, 2nd ed., Addison-Wesley Publishing Company, Reading, MA, 1994.

[9] P. Hilton, J. Pedersen, Catalan numbers, their generalization, and their uses, Math. Intelligencer, 13 (1991), 64-75; Available online at http: //dx.doi.org/10.1007/BF03024089.

[10] D. A. Klarner, Correspondences between plane trees and binary sequences, J. Combinatorial Theory, 9 (1970), 401-411.

[11] T. Koshy, Catalan Numbers with Applications, Oxford University Press, Oxford, 2009.

[12] C. -H. Lin, Some combinatorial interpretations and applications of FussCatalan numbers, ISRN Discrete Math., 2011 (2011), Article ID 534628, 8 pages; Available online at http://dx.doi.org/10.5402/2011/534628.

[13] F. -F. Liu, X.-T. Shi, F. Qi, A logarithmically completely monotonic function involving the gamma function and originating from the Catalan numbers and function, Glob. J. Math. Anal., 3 (2015), 140-144; Available online at http://dx.doi.org/10.14419/gjma.v3i4.5187.

[14] D. -Z. Liu, C. -W. Song, Z. -D. Wang, On explicit probability densities associated with Fuss-Catalan numbers, Proc. Amer. Math. Soc., 139 (2011), 3735-3738; Available online at http://dx.doi.org/10.1090/ S0002-9939-2011-11015-3.

[15] M. Mahmoud, F. Qi, Three identities of Catalan-Qi numbers, Mathematics, 4 (2) (2016), Article 35, 7 pages; Available online at http: //dx.doi.org/10.3390/math4020035.

[16] J. McCarthy, Catalan numbers. Letter to the editor: "Catalan numbers, their generalization, and their uses" [Math. Intelligencer, 13 (1991), 64-75] by P. Hilton and J. Pedersen, Math. Intelligencer, 14 (1992), 5. 
[17] D. S. Mitrinović, J. E. Pečarić, A. M. Fink, Classical and New Inequalities in Analysis, Kluwer Academic Publishers, DordrechtBoston-London, 1993; Available online at http://dx.doi.org/10.1007/ 978-94-017-1043-5.

[18] W. Młotkowski, Fuss-Catalan numbers in noncommutative probability, Doc. Math., 15 (2010), 939-955.

[19] W. Młotkowski, K. A. Penson, K. Życzkowski, Densities of the Raney distributions, Doc. Math., 18 (2013), 1573-1596.

[20] I. Pak, Catalan Numbers Page, available online at http://www.math. ucla.edu/ pak/lectures/Cat/pakcat.htm.

[21] J. H. Przytycki, A. S. Sikora, Polygon dissections and Euler, Fuss, Kirkman, and Cayley numbers, J. Combin. Theory Ser. A, 92 (2000), 68-76; Available online at http://dx.doi.org/10.1006/jcta.1999.3042.

[22] F. Qi, Asymptotic expansions, complete monotonicity, and inequalities of the Catalan numbers, ResearchGate Technical Report, (2015); Available online at http://dx.doi.org/10.13140/RG.2.1.4371.6321.

[23] F. Qi, B. -N. Guo, Logarithmically complete monotonicity of Catalan-Qi function related to Catalan numbers, Cogent Math., (2016), 3:1179379, 6 pages; Available online at http://dx.doi.org/10.1080/23311835. 2016.1179379 .

[24] F. Qi, Some properties and generalizations of the Catalan, Fuss, and Fuss-Catalan numbers, ResearchGate Research, (2015); Available online at http://dx.doi.org/10.13140/RG.2.1.1778.3128.

[25] F. Qi, Two product representations and several properties of the FussCatalan numbers, ResearchGate Research, (2015); Available online at http://dx.doi.org/10.13140/RG.2.1.1655.6004.

[26] F. Qi, C. -P. Chen, A complete monotonicity property of the gamma function, J. Math. Anal. Appl., 296 (2004), 603-607; Available online at http://dx.doi.org/10.1016/j.jmaa.2004.04.026.

[27] F. Qi, S. -X. Chen, Complete monotonicity of the logarithmic mean, Math. Inequal. Appl., 10 (2007), 799-804; Available online at http://dx.doi. org/10.7153/mia-10-73. 
[28] F. Qi, B. -N. Guo, Complete monotonicities of functions involving the gamma and digamma functions, RGMIA Res. Rep. Coll., 7 (2004), 63-72; Available online at http://rgmia.org/v7n1.php.

[29] F. Qi, M. Mahmoud, X. -T. Shi, F. -F. Liu, Some properties of the Catalan-Qi function related to the Catalan numbers, SpringerPlus, (2016), 5:1126, 20 pages; Available online at http://dx.doi.org/10. $1186 / \mathrm{s} 40064-016-2793-1$.

[30] F. Qi, X. -T. Shi, P. Cerone, A unified generalization of the Catalan, Fuss, Fuss-Catalan numbers and Catalan-Qi function, ResearchGate Working Paper, (2015); Available online at http://dx.doi.org/10.13140/RG.2. 1.3198 .6000 .

[31] F. Qi, X. -T. Shi, F. -F. Liu, An exponential representation for a function involving the gamma function and originating from the Catalan numbers, ResearchGate Research, (2015); Available online at http://dx.doi.org/ 10.13140/RG.2.1.1086.4486.

[32] F. Qi, X. -T. Shi, F. -F. Liu, An integral representation, complete monotonicity, and inequalities of the Catalan numbers, ResearchGate Technical Report, (2015); Available online at http://dx.doi .org/10.13140/RG.2. 1.3754 .4806 .

[33] F. Qi, X. -T. Shi, F. -F. Liu, Several formulas for special values of the Bell polynomials of the second kind and applications, ResearchGate Technical Report, (2015); Available online at http://dx.doi.org/10.13140/RG. 2.1.3230.1927.

[34] F. Qi, X. -T. Shi, M. Mahmoud, F. -F. Liu, Schur-convexity of the Catalan-Qi function, ResearchGate Technical Report, (2015); Available online at http://dx.doi.org/10.13140/RG.2.1.2434.4802.

[35] F. Qi, X. -J. Zhang, W. -H. Li, An integral representation for the weighted geometric mean and its applications, Acta Math. Sin. (Engl. Ser.), 30 (2014), 61-68; Available online at http://dx.doi.org/10. $1007 /$ s10114-013-2547-8.

[36] F. Qi, X. -J. Zhang, W. -H. Li, Lévy-Khintchine representation of the geometric mean of many positive numbers and applications, Math. Inequal. Appl., 17 (2014), 719-729; Available online at http://dx.doi.org/10. 7153/mia-17-53. 
[37] F. Qi, X. -J. Zhang, W. -H. Li, Lévy-Khintchine representations of the weighted geometric mean and the logarithmic mean, Mediterr. J. Math., 11 (2014), 315-327; Available online at http://dx.doi.org/10.1007/ s00009-013-0311-z.

[38] R. L. Schilling, R. Song, Z. Vondraček, Bernstein Functions-Theory and Applications, 2nd ed., de Gruyter Studies in Mathematics 37, Walter de Gruyter, Berlin, Germany, 2012; Available online at http://dx.doi. org/10.1515/9783110269338.

[39] X. -T. Shi, F. -F. Liu, F. Qi, An integral representation of the Catalan numbers, Glob. J. Math. Anal., 3 (2015), 130-133; Available online at http://dx.doi.org/10.14419/gjma.v3i3.5055.

[40] R. Stanley, E. W. Weisstein, Catalan number, From MathWorld-A Wolfram Web Resource; Available online at http://mathworld.wolfram. com/CatalanNumber.html.

[41] C. Stump, q, t-Fuß-Catalan numbers for complex reflection groups, 20th Annual International Conference on Formal Power Series and Algebraic Combinatorics, 2008, 295-306, Discrete Math. Theor. Comput. Sci. Proc., AJ, Assoc. Discrete Math. Theor. Comput. Sci., Nancy, 2008.

[42] C. Stump, q, t-Fuß-Catalan numbers for finite reflection groups, J. Algebraic Combin., 32 (2010), 67-97; Available online at http://dx.doi. org/10.1007/s10801-009-0205-0.

[43] I. Vardi, Computational Recreations in Mathematica, Addison-Wesley, Redwood City, CA, 1991.

[44] D. V. Widder, The Laplace Transform, Princeton Mathematical Series 6 , Princeton University Press, Princeton, N. J., 1941.

[45] Wikipedia, Fuss-Catalan number, From the Free Encyclopedia; Available online at https://en.wikipedia.org/wiki/Fuss-Catalan_number. 